\title{
LA ARITMÉTICA DE BOECIO Y LA RITMOMAQUIA: TEORÍA Y PRÁCTICA DEL JUEGO MEDIEVAL DE LOS SABIOS
}

\author{
JOSÉ M. NÚÑEZ ESPALLARGAS ${ }^{1}$
}

Resumen: En este estudio se analizan los fundamentos teóricos de la ritmomaquia, el primer juego creado y diseñado expresamente para la enseñanza y aprendizaje de la matemática en la cultura occidental. Se trataba de una compleja competición numérica practicada originariamente en las escuelas monacales centroeuropeas del medievo. Su propósito inicial era la adquisición teórica y práctica de la aritmética, aunque sus fines trascendían esa función al presentar un modelo didáctico que permitía constatar la armonía matemática que regía el universo. Era también un juego practicado por la nobleza culta de la época, que buscaba en esta competición una manera de alcanzar adiestramiento intelectual. Así se comprende que los germanos la denominaran «el juego de la batalla de números» (das Zahlenkampfspiel) y los anglosajones «el juego de los sabios» (the Philosophers' Game). Tenemos bastantes lagunas sobre su origen derivadas de las escasas referencias medievales conservadas y que han ayudado a crear una cierta aureola dehermetismo. La mayor parte de información proviene de descripciones renacentistas, época en la que el juego alcanzó un efímero apogeo, para luego caer bruscamente en el olvido. En este trabajo se aporta una interpretación sobre la generación de los números que intervienen en el juego, basada directamente en la utilización de la terminología latina del texto de Boecio, que ofrece una explicación que armoniza perfectamente con los contenidos y objetivos de la matemática medieval. También se discuten las variantes de las reglas del juego y se presentan argumentos que ayudan a explicar su decadencia y desaparición.

Palabras clave: Boecio; Aritmética; Ritmomaquia; Juego matemático.

Abstract: This research analyses the theoretical bases of rithmomachia, a complex numerical competition originally practiced in medieval Central European monastic schools. The initial objective of the game was the theoretical and practical acquisition of arithmetic, though this aim was surpassed with the presentation of a reduced model of the mathematical harmony which governs the universe. The game was also played by the nobility of the period who used it as a source of intellectual training. This study proposes an interpretation of the generation of the numbers that intervene in this game; the interpretation is based on the use of the Latin terminology of Boetius' text and offers an explanation that is wholly compatible with the

'Catedrático de la Facultad de Formación del Profesorado, Universidad de Barcelona. 2003

Fecha de recepción del artículo: marzo 2003. Fecha de aceptación y versión final: diciembre

«Anuario de Estudios Medievales», 34/1 (2004), pp. 279-306.- ISSN 0066-5061. 
contents and objectives of medieval mathematics. Also discussed are the variations in the game's rules and the possible reasons for the game's decline and disappearance.

Keywords: Boetius; Aritmethic; Rithmomachia; Mathematical game.

\section{SUMARIO}

1. Sobre el origen y las fuentes de la ritmomaquia.- 2. Auge y decadencia de la ritmomaquia.- 3. Boecio y la decadencia de la aritmética en el Medievo.- 4. Los fundamentos matemáticos de la ritmomaquia.- 5 . Sobre las reglas de juego y sus variantes.- 6 . Conclusiones.

\section{SOBRE EL ORIGEN Y LAS FUENTES DE LA RITMOMAQUIA}

Los orígenes de la ritmomaquia son inciertos. BoISSIÈRE (1556), el autor del manual clásico más importante sobre ritmomaquia atribuyó el origen de este juego a los caldeos, pero no aportó prueba alguna en favor de su aserto $^{2}$. Para la mayoría de los antiguos textos de ritmomaquia la invención se sitúa en el ámbito de la Grecia clásica y, más concretamente, en el entorno de la escuela pitagórica, señalando incluso a su fundador como el inventor del juego ${ }^{3}$. La misma palabra «ritmomaquia» parece apoyar esta opinión; es un término que reúne dos raíces griegas: la primera es una deliberada y hábil combinación de «arithmos» $\mathrm{y}$ «rhythmos», es decir, de número y de ritmo, y podríamos interpretarla como «proporción de números»; la segunda, «machia», designa lucha, batalla. Además del nombre, de clara raigambre griega, los fundamentos teóricos del juego se inspiran en la concepción que los pitagóricos tenían del mundo y de los números que eran su armazón. Pero, a pesar de estas atribuciones, no existe ninguna referencia escrita del juego en los antiguos textos, ni griegos ni romanos, ni tampoco se han encontrado vestigios arqueológicos (un tablero o una pieza, por ejemplo) del mismo.

Algún autor contemporáneo (BORST 1986), al observar similitudes entre la colocación de las piezas en el tablero y sus movimientos con las estrategias organizativas y de ataque de las legiones romanas, ha sugerido la

2 "...à esté extracte d'un ancien livre Chaldée..." (BoISSIÈRE, 1556, p. 41 v.).

${ }^{3}$ Véase, por ejemplo, la referencia en el manuscrito 14065 de la Bibliothèque Nationale de París (Olim S. Germani 600, fonds St. Germain 1095) s. XIII, s. XI ex vol. XII in fols. 1-5 v. ODONIS, Rithmomachia: Rithmimachia grece numerorum pugna exponitur.... También encontramos esta atribución en el título de los tratados de BAROZZI (1572): Il nobilisimo et antiquissimo giuco pythagoreo..., y de BOISSIËRE (1556): Nobilissimus et antiquissimus ludus Pythagoreus....

«Anuario de Estudios Medievales», 34/1 (2004), pp. 279-306.- ISSN 0066-5061. 
posibilidad de que fuera una invención dentro del ámbito cultural griego tardío y, más concretamente, del Imperio Bizantino. Pero, tampoco se han encontrado referencias documentales o materiales que prueben esta hipótesis.

Pero todas las pruebas existentes nos sitúan el origen del juego en una época posterior. Las referencias conocidas de la ritmomaquia no van más allá del siglo XI. Siguiendo a SMITH (1970) habría cuatro fuentes fundamentales del juego, de cada una de las cuales existen varias copias conservadas en diferentes bibliotecas. Las copias son, en algunas ocasiones, reproducciones fieles de un mismo original, en otras, el texto básico es ampliado con anexos que describen ejemplos de armonías. La versión más antigua parece tener como autor al monje Hermannus Contractus, cuya fecha de muerte se remonta al año 1054. Es conocida como Hermanni ritmomachia y de ella se conservan tres manuscritos ${ }^{4}$. Otra versión es la del benedictino Accolytus del 1077, que sitúa la práctica del juego en el monasterio de Würzburg; es la denominada Ritmomaquia Wirceburgensis y de la que se conocen siete copias manuscritas. La tercera fuente (Odonis ritmomaquia), también del siglo XI, se atribuye a un monje de nombre Odo y se han localizado dos copias ${ }^{6}$. Finalmente tenemos la escrita por el monje Fortolfus (Fortolfi ritmomachia), de la que sólo conocemos un manuscrito ${ }^{7}$. También podemos incluir en esta relación de fuentes primarias un dibujo del tablero del juego sin texto explicatorio conservado en Karlsruhe ${ }^{8}$.

Señalemos que, a pesar de su supuesto origen griego, no ha aparecido ningún manuscrito en lengua griega, ni tampoco referencia alguna al juego de la ritmomaquia en la Europa Oriental. Tampoco se ha encontrado ninguna

${ }^{4}$ Uno en la München Bibliothek (14836 Emmer. K.6 s.XI in 8 fol.3 v - 4 v), otro en la Bibliothèque Nationale de París (7185 Olim P. Pithou, J. de Thou 283, Colbert 4001, Regius 5365 s.XII ex vol.XIII fol. $107 \mathrm{r}$ ) y el tercero en la Biblioteca Vaticana (3101 a Benedicto accolyto monasterii S. Arsacii a.1077 scriptus in 4 fol. 28 r).

${ }^{5}$ Se localizan en las siguientes bibliotecas: München Bibliothek (14836 Emmer. K.6 s.XI in 8 fol. $4 \mathrm{v}-6 \mathrm{v}$ ), Bodleian Library de Oxford (Rawlinson C.270 s.XII in 8 fol. $20 \mathrm{r}-22 \mathrm{r}$ ) Bibliothèque National de Paris (7185 Olim P. Pithou, J. de Thou 283, Colbert 4001, Regius 5365 s.XII ex vol. XIII fol. $107 \mathrm{v}-108 \mathrm{v})$, (14065 Olim S. Germani 600, fonds St. Germain 1095 s.XIII, s.XI ex vol. XII fol. 5 v-6 v) y (15119 Olim S. Victor 533 s.XII fol. 9 r-12 r) y Biblioteca Vaticana (3101 Benedicto accolyto monasterii S. Arsacii a. 1077 in 4 fol. 3 r-5 v) y (Christ. 598 s.XI).

${ }^{6}$ Bibliothèque National de Paris (14065 Olim S. Germani 600, fonds S. Germain 1095 s.XIII, s.XI ex vol. XII fol.1 r-5 v) y Österreichische Nationalbibliothek de Viena (2503 s.XIII in 8 fol. $49 \mathrm{r}-57 \mathrm{v}$ )

${ }^{7}$ Breslau Bibliothek 54 s.XII.

${ }^{8}$ Badischen Landesbibliothek Karlsruhe 504, fol. 87 r.

«Anuario de Estudios Medievales», 34/1 (2004), pp. 279-306.- ISSN 0066-5061. 
noticia en los archivos medievales del extremo occidental de Europa. Por el contrario y como acabamos de describir, los documentos más antiguos han sido localizados exclusivamente en la Europa Central y relacionados todos ellos con la vida monástica. Parece pues plausible, situar en ese entorno su origen. Observemos que las primeras referencias están relacionadas con la expansión de las escuelas monacales y catedralicias en las que se impartía la aritmética de Boecio integrada en el sistema del quadrivium.

Hagamos notar, también, que todas las fuentes conocidas, si bien sirven para documentar la existencia del juego, nos ayudan poco para el conocimiento de sus reglas, son textos breves ${ }^{9}$, y su redacción es frecuentemente oscura, dando las reglas básicas e incluso el valor de las piezas por conocidas, preocupándose únicamente en ofrecer un conjunto de soluciones a problemas concretos, una especie de recetarios expresados muchas veces en forma críptica. Todo ello ha dado pie a pensar (BORST, 1986) que esa manera hermética que presentan los textos medievales conservados, que los aleja de lo que podrían ser manuales o introducciones didácticas al juego, es deliberada, para que así quedara la práctica de la ritmomaquia fuera del alcance del público, reservándola sólo a los iniciados, pertenecientes, generalmente, al círculo de los monasterios y sus aledaños.

Tenemos referencias indirectas que corroboran este uso didáctico de la ritmomaquia. Juan de Salisbury, uno de los más cultos pensadores escolásticos del siglo XII, hace un encendido encomio de la ritmomaquia como fuente de entretenimiento y de provecho intelectual ${ }^{10}$. Un siglo más tarde, el franciscano Roger Bacon, recomienda el juego a los estudiantes de matemáticas como una ayuda para el aprendizaje de la aritmética de Boecio ${ }^{11}$. Incluso Tomás Moro, ya en pleno Renacimiento, cuando describe las ejemplares costumbres de los habitantes de «Utopía» durante las veladas nos habla de que desechan los juegos vanos y perniciosos practicando sólo una

${ }^{9} \mathrm{La}$ Odonis rithmomachia la fuente más extensa tiene 10 páginas en el manuscrito 14065 de la Bibliothèque National de Paris y casi 18 (de menor tamaño) en el manuscrito 2503 de la Österreichische Nationalbibliothek de Viena; mientras que la Hermanni rithmomachia ocupa un único folio, tanto en el manuscrito 14836 de la München Bibliothek como en el 7185 de la Bibliothèque National parisina y en el 3101 de la Biblioteca Vaticana.

${ }^{10} \mathrm{~J}$. SAlisBURY, Policraticus. De nugis curialium et vestigiis philosophorum. Lib. I , cap. 5.(c.1159). (Existe traducción inglesa por John DICKINSON, The Statesman's Book of John of Salisbury. New York, Alfred A. Knopf, 1927, pp. 258-263).

${ }^{11}$ R. BACON, Opus majus, Lib. I, cap. 3. (c. 1267). (Existe una traducción inglesa íntegra por Robert B. BURKE, The Opus Majus of Roger Bacon (2 vol.) University of Pennsylvania Press, 1928.)

«Anuario de Estudios Medievales», 34/1 (2004), pp. 279-306.- ISSN 0066-5061. 
especie de «batalla de números» y otro juego que enfrenta vicios contra virtudes ${ }^{12}$.

A través de estas alusiones obtenemos una imagen coincidente de como era percibida la ritmomaquia por sus contemporáneos: era un juego que permitía el aprendizaje agradable de la aritmética y, especialmente, de la manera como era expuesta por Boecio, pero también era un entretenimiento intelectual provechoso a los ojos de la religiosidad católica, tan crítica, por otra parte, con cualquier otro tipo de pasatiempos, pues, incluso el ajedrez, que no estaba explícitamente vetado, tampoco estaba incluido en la categoría de entretenimientos virtuosos.

Cuando se conoce el juego y sus reglas se puede comprender perfectamente porque la ritmomaquia es útil en la enseñanza de la aritmética. Pero, lo que ya no es tan evidente es la catalogación de juego virtuoso que todos estos autores le atribuyen. Si consideramos los juegos de dados, es claro que son un mero entretenimiento que no ejercita la mente y mucho menos el espíritu; por consiguiente su práctica no aporta ningún beneficio al buen cristiano. Pero el rechazo del ajedrez, un juego intelectual, con el que la ritmomaquia guarda claras similitudes, no se entiende tan fácilmente. La explicación de esta diferente consideración debemos buscarla, no en la tipología de juego, sino en sus diferentes objetivos. En el ajedrez los jugadores persiguen «dar mate» a una figura, el rey, propósito que suelen alcanzar tras «matar» a otras figuras que se interponen en el camino. Es cierto, que también en la ritmomaquia se pueden «capturar» piezas, pero el objetivo del juego no es el de «matar», sino el de situar una serie de piezas de modo que se consiga uno de los tres tipos de proporciones numéricas establecidos por Boecio. De este modo el ganador pretende alcanzar, al colocar las fichas en alguna de estas «ordenaciones», los grados de armonía que rigen en el universo, lo que implícitamente supone un reconocimiento de la sabiduría de su Creador. Se establece una diferencia entre lo que es un juego profano, como los dados o el ajedrez, que sólo pretenden entretener, de un juego, que a través del ejercicio intelectual, se propone un objetivo religioso.

\footnotetext{
${ }^{12}$ «Allí practican la música o se entretienen conversando. Desconocen totalmente los dados o cualquier otro juego vano y pernicioso, aunque si juegan a dos pasatiempos parecidos al ajedrez. Uno consiste en una batalla de números, en que unos se comen a otros; y el otro en un conflicto armado de los vicios contra las virtudes» T. MORE, Utopia. De optimo reipublicae statu. Lib. II, cap. 4 (1516). (Fragmento tomado de la traducción española de Andrés Vázquez de Prada, Utopía, Madrid, Rialp, 1991, p. 112). 
En resumen, de todas las fuentes directas e indirectas que conocemos parece deducirse que la ritmomaquia fue concebida a mediados del siglo XI en el ámbito monacal del sur de Alemania. El propósito del juego sería el de ilustrar a los alumnos de las escuelas monacales en la teoría de números de Boecio. Con la práctica del mismo se facilitaría la memorización de las relaciones entre los números y se adquiriría destreza en el cálculo. A finales del siglo XI y durante el XII se extendería por todo el sur de Alemania y Francia. Entre finales del XII y principios del XIII salta el Canal de la Mancha y se difunde por Inglaterra.

\section{AUGE Y DECADENCIA DE LA RITMOMAQUIA}

Con la aparición de la imprenta las reglas de la ritmomaquia pudieron difundirse con más facilidad, rebasando así el ámbito de influencia de los monasterios, y, de este modo, el juego alcanzó entonces su máxima expansión. Se publicaron auténticos manuales, que nos han permitido conocer mejor el juego y sus variantes, aclarando muchos aspectos oscuros de las reglas que no pueden deducirse de las escuetas informaciones proporcionadas por los antiguos manuscritos. El más antiguo texto impreso conocido es debido al clérigo, futuro obispo de Durham, John Shirwood. Se trata de una muy sucinta introducción al juego y a sus reglas básicas. Fue editado en Roma en 1480 y dedicado a su soberano o, lo que equivaldría a decir, a la nobleza. El siguiente, a pesar también de su brevedad, está considerado como el primer manual completo sobre el tema. Es en realidad un apéndice a las obras de aritmética y de música de Boecio y de Jordanus Nemorarius. Aparece publicado en París dos años antes del descubrimiento de América y es debido a la pluma del profesor Jacobus Faber Estapulensis (Jacques Lefèvre d'Etables). Está escrito en forma dialogada siguiendo la tradición pitagórica y orientado a sus estudiantes de la Universidad de Paris. Estos dos primeros textos sobre ritmomaquia ya muestran perfectamente los dos tipos de público al que iban dirigidos: la nobleza o elite culta y a los estudiantes de matemáticas.

En 1554 Claudius Buxerius (Claude de Boissière) publica en París un texto de ritmomaquia que iba a gozar de gran aceptación. Fue también escrito, como el de Faber, con intención de que sirviera de ayuda a los alumnos de la universidad parisina. Dos años más tarde, el mismo Boissière, da a la imprenta una traducción francesa, constituyendo el primer manual del juego 
publicado en una lengua vernácula, lo que contribuyó, sin duda, a su mayor difusión. En uno de los ejemplares conocidos de la obra se localizó un anuncio de la venta de tableros y de piezas del juego en un establecimiento de la ciudad (SMITH 1911). Este dato prueba que llegaron a manufacturarse juegos completos, aunque no se ha conservado ninguna prueba material sobre el juego, ni de época medieval ni del Renacimiento. Es el manual más detallado de los publicados en esta época y en él, su autor, se propone extender la práctica de la ritmomaquia explicando con la mayor claridad posible las reglas del juego, aclarando así las múltiples situaciones confusas que se derivaban de la lectura de otros textos y que hacían poco accesible el juego al público interesado ${ }^{13}$. A nuestro juicio la obra de Boissière es el más clarificador de los textos clásicos; no carece de algunos puntos oscuros, pero en conjunto es la obra de referencia básica de los estudiosos sobre el tema. Nosotros, a la hora de comentar las reglas del juego, nos referiremos a él con preferencia.

En estos años (1563), en Londres, se publica, en inglés, el texto de William Fulker y Rafe Lever, que será la obra más divulgada en Inglaterra. Poco tiempo después, en 1572, en Venecia y en lengua italiana, Francesco Barozzi publica un nuevo tratado, que si bien no tiene la calidad expositiva del de Boissière, llegó a alcanzar una gran difusión en Centroeuropa. Señalaremos, también, la existencia de un manuscrito debido a la pluma de Abraham Ries, hijo segundo del famoso «maestro de cálculo» (Rechenmeister) Adam Ries y datado en 1562, que constituye el primer texto sobre el tema en lengua alemana ${ }^{14}$. Ries escribe su obra ignorando los textos franceses, y se inspira directamente en antiguos manuscritos de los monasterios alemanes y en el manual de John Shirwood (MEBBEN 1996). Las tres últimas obras citadas están dedicadas a los respectivos monarcas o protectores.

Esta eclosión de manuales de ritmomaquia y, por tanto, de interés por este juego no se explica únicamente por la difusión de la imprenta, aunque, sin duda, este invento la favoreció. En el siglo XV y XVI, era frecuente que muchos profesores en las universidades simultanearan las enseñanzas científicas con las filosóficas y teológicas como una manera de enfocar

\footnotetext{
${ }^{13}$ "Au iourd huy toutes fois n'est en uisage à cause des enigmatiques interpretations ou iusques à present il a esté caché \& obscurcy» (BOISSIÈRE, 1556, p. 4 v.).

${ }^{14}$ Dresde Bibliothek C-433.

«Anuario de Estudios Medievales», 34/1 (2004), pp. 279-306.- ISSN 0066-5061.
} 
globalmente la obra de Dios ${ }^{15}$. No es de extrañar pues, que, en este contexto, la concepción de un universo ordenado y jerarquizado tuviera en el estudio de la teoría de números de Boecio una base sólida. La ritmomaquia ayudaría a adiestrar las mentes en esta creencia de que las proporciones y relaciones entre los números regían el mundo.

Pero esta visión del mundo dominante durante el Medievo y el primer Renacimiento empezó a zozobrar ya a finales del siglo XVI. Los datos experimentales cada vez más precisos que se conseguían con el telescopio, recién inventado, hacían que la regularidad del cosmos no fuera tan «exacta» como predecían las teorías ptolemaicas. Las matemáticas de Boecio, centradas en el estudio de los números y sus relaciones, comenzaban a quedar desplazadas tras la introducción del álgebra, que amplificaba las posibilidades de la aritmética. Pero, incluso, desde la misma escolástica irrumpían voces en contra de la concepción aristotélica del universo. El más duro crítico del aristotelismo, el francés Pierre Ramus, que además de profesor de dialéctica lo fue también de matemáticas, en su obra Scholarum mathematicarum ${ }^{16}$, sobre la enseñanza de las matemáticas, ignora completamente la ritmomaquia. Este hecho resulta sorprendente, dado que era contemporáneo de Boissière y docente como él de la Universidad de París. Pero si estudiamos la obra de Ramus veremos que incluye un feroz ataque a la visión pitagórica que inspiraba la obra matemática de Boecio. Para Ramus la importancia de los números no radica en la belleza de sus proporciones y de sus relaciones con la geometría, sino en el uso que de ellos se hace en el comercio, en la industria y en las artes liberales en genera ${ }^{17}$. La enseñanza de la matemática debía enfocarse pues, no hacia la contemplación de las proporciones y las armonías, sino hacia la utilidad. De este modo, se propiciaba una separación gradual de la enseñanza de la matemática de la filosofía y de la teología. La consolidación de esta tendencia se evidencia con la creación de cátedras de matemáticas en las universidades centroeuropeas ya a fines del siglo XVI.

\footnotetext{
${ }^{15}$ «La contemplación y el estudio del cosmos ordenado, jerarquizado, así como de las leyes de la naturaleza, eran tanta parte de la contemplación y el estudio de Dios como la teología y la p. 364).

${ }^{16} \mathrm{P}$. RAMUS, Scholarum mathematicarum libri unus et triginta, Basilea, 1569.

${ }^{17}$ Véase J.M. NúÑEZ; A. GRAU, Petrus Ramus (1515-1572) y su concepción renovadora de la enseñanza de las matemáticas, «Revista de Educación», 318 (1999), pp. 165-173.

«Anuario de Estudios Medievales», 34/1 (2004), pp. 279-306.- ISSN 0066-5061.
} 
Durante el siglo XVII la popularidad de la ritmomaquia decrece rápidamente. El que había sido el juego de los sabios, de los nobles cultos y de los estudiantes de matemáticas cae en el más absoluto olvido. Es muy sintomático que, una figura como Leibnitz (1646-1716), buen conocedor del saber filosófico y matemático de su época, del juego sabía sólo su nombre, ignorando totalmente sus reglas (MEBBEN 1996). Si bien es cierto que en el siglo XVII ya no se publica ningún texto de ritmomaquia dirigido a los estamentos que en los siglos anteriores habían sido sus entusiastas seguidores, no sería exacto afirmar que desaparecen totalmente las referencias. Las escasas citas se sitúan en un ámbito diferente: el de los cultivadores de los juegos de entretenimiento, que en su práctica no pretenden alcanzar ningún objetivo útil o trascendente. La referencia paradigmática es la obra de G. Selenus, Das Schach oder König-Spiel (1616), un libro dedicado al ajedrez, el juego que había ocupado el lugar de la ritmomaquia en el solaz de las clases cultas. En él, y a modo de apéndice, se incluye un apartado dedicado a la ritmomaquia, que es prácticamente una traducción del texto de Barozzi.

Esta presencia en el manual de ajedrez, clásico por excelencia, hizo que otros textos de ajedrez, todos ellos del área lingüística alemana, con mayor o menor extensión, dedicaran algunas páginas a la «batalla de números» tratándola como una variante numérica del ajedrez. Muy probablemente esta pervivencia residual hizo que la memoria del juego se mantuviera. A mediados del siglo XIX un afamado profesor de matemáticas y experto jugador de ajedrez de nombre Adler intentará recuperar los aspectos educativos del juego en su programa escolar de 1852, aunque sin éxito. En 1917, Jahn, párroco y también profesor de matemáticas, lo volvió a intentar con sus alumnos, pero tampoco obtuvo buenos resultados (MEBBEN 1996). Si bien estos intentos por recuperar la finalidad didáctica de la ritmomaquia fracasaron, al menos sirvieron para que una parte de la comunidad científica fijara su atención en el juego. Durante la pasada centuria se han realizado diversos estudios encaminados a descubrir el origen y a desentrañar las reglas del juego. Algunas investigaciones anglosajonas han ayudado a clarificar las fuentes, pero, especialmente han sido autores germanos, como Borst, Illmer y Mebben los que han llevado a cabo trabajos más extensos y concienzudos. 


\section{BOECIO Y LA ENSEÑANZA DE LA ARITMÉTICA EN EL MEDIEVO}

Para comprender el valor asignado a las piezas y las reglas básicas de la ritmomaquia es necesario conocer los rasgos generales de la enseñanza de la aritmética en la Edad Media y más concretamente la teoría de las proporciones numéricas desarrollada por Boecio (c.480-524) en su De Institutione Arithmeticae $^{18}$.

Es bien sabido que la enseñanza en las universidades medievales se organizaba en torno a dos grupos de materias: el trivium y el quadrivium. La enseñanza del quadrivium (aritmética, geometría, música y astronomía) reunía la formación científica que complementaba el núcleo esencial de la enseñanza medieval que estaba constituido por el trivium (gramática, dialéctica y retórica). El hecho de que cuatro disciplinas constituyeran la base de la formación científica medieval viene perfectamente justificado por Boecio. Para este pensador el objeto de todo estudio debe ser la esencia (esentia), la cual divide en continua y discontinua. La esencia continua es aquella que no es divisible en unidades más pequeñas y la discontinua es la que está constituida por elementos menores. Dentro de la esencia continua propone estudiar las magnitudes (magnitudines) que tienen carácter inmóvil, lo que constituye la geometría, o móvil, que caracteriza a la astronomía. Con respecto a la esencia discontinua, la base estará en la multiplicidad (multitudo) y puede dividirse su estudio entre la que tiene existencia en si misma, como los diferentes números, y que constituye la aritmética, y la que tiene su existencia condicionada a la anterior, propiedad que define a la música ${ }^{19}$.

Veamos ahora, también muy esquemáticamente, el tratamiento que Boecio da a la multiplicidad, es decir a los números y a sus relaciones mutuas. Precisemos antes, que la teoría desarrollada por Boecio no es totalmente originaria suya, tiene sus raíces en la escuela pitagórica y su referente más próximo lo encontramos en la aritmética del matemático griego del siglo II

\footnotetext{
${ }^{18}$ Disponemos de varias ediciones modernas bilingües de las Institutione Arithmeticae. Por ejemplo, la versión francesa debida a Jean-Yves Guillaumin (Paris, Les Belles Lettres, 1995) y la traducción española de María A. Sánchez Manzano (León, Universidad de León, 2002).

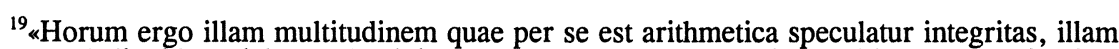
uero quae ad aliquid musici modulaminis temperamenta pernoscunt, immobilis uere magnitudinis geometria notitiam pollicetur, mobilis uero scientiam astronomiae disciplinae peritia vindicat» (BOETHIUS, De Institutione Arithmetica, 1, 1, 4).
} 
Nicómaco de Gerasa ${ }^{20}$. Pero, dado que durante toda la Edad Media hasta el Renacimiento, que comienzan a conocerse en Europa las versiones y comentarios árabes de los antiguos textos clásicos, la enseñanza de la aritmética se basa única y exclusivamente en el texto de Boecio, nuestras referencias a la teoría de números se centraran en este autor.

Al tratar las relaciones entre los números, Boecio comienza por plantear que dos números pueden ser entre si iguales (aequalis) o desiguales (inaequalis). La igualdad, por su carácter, es indivisible en categorías y no recibe ningún otro nombre que el mismo que tiene la cantidad con la que se compara. La desigualdad, en cambio, tiene una primera división en dos apartados: un número puede ser desigual a otro por exceso y entonces hablaremos de la categoría de mayor (maioris); también puede ser desigual por defecto, en cuyo caso tendremos la categoría de menor (minoris).

Cada una de estas categorías principales de la desigualdad se divide en cinco subcategorías, dependiendo de la relación que pueda establecerse entre los dos números. Por lo que se refiere a los maioris la subdivisión es la siguiente:

Múltiplo (multiplex) cuando contiene un número entero de veces el número con el que se compara. Se denomina doble (duplus), triple (triplus), cuádruple (quadruplus), dependiendo de las veces que lo contenga. Ejemplo: 8 es quadruplus de 2 , pues $8=4 * 2$.

Superparticular (superparticularis) cuando contiene al número con el que se compara una vez entero más una parte del mismo. Recibe un nombre que indica el denominador de la fracción unitaria que se añade. Así, si se suma un medio se llamará sesquialterus; si es un tercio, sesquitertius; etc. Ejemplo: 8 es sesquitertius de 6 , pues $8=(1+1 / 3) 6$.

Superpartiente (superpartiens) cuando contiene al número con el que se compara una vez entero más varias partes del mismo. La denominación hará referencia al numerador de la fracción propia que se suma, si ésta es dos tendremos superbipartientes; si es tres, supertripartientes; etc. Ejemplo: 7 es supertripartientes de 4 , pues $7=(1+3 / 4) 4$.

Las dos subdivisiones siguientes Boecio las denomina especies complejas, para distinguirlas de las dos anteriores que eran especies simples. La razón es sencilla de entender: las especies complejas se obtienen de la

${ }^{20}$ «De Institutione Arithmetica es como una versión de la Aritmética de Nicómaco» H. WIELEITNER, Historia de las matemáticas, Barcelona, Labor, 1928, p. 46.

«Anuario de Estudios Medievales», 34/1 (2004), pp. 279-306.- ISSN 0066-5061. 
combinación de múltiplos con superparticulares y superpartientes. Tendremos así:

Múltiplos superparticulares (multiplex superparticularis) serán los números que contienen a otro en su totalidad más de una vez y, además, una parte de él. El nombre hará alusión tanto al múltiplo como al denominador de la fracción unitaria que se añade. Ejemplo: 16 es triplus sesquiquintus de 5, pues $16=(3+1 / 5) 5$.

Múltiplos superpartientes (multiplex superpartiens) serán los números que contienen a otro en su totalidad más de una vez y, además, varias partes de él. La denominación se referirá tanto al múltiplo como al superpartiente que interviene. Ejemplo: 29 es quintus superquadripartientes de 5, pues 29 $=(5+4 / 5) 5$.

Por lo que respecta a la relación de un número con cantidades menores (minoris), también Boecio establece cinco subclases que son las inversas de las maioris. Tienen las mismas denominaciones, pero añadiendo el prefijo $s u b$. Así, invirtiendo los ejemplos anteriores tendremos: 2 es submultiplus de 8, 6 es subsesquitertius de 8, 4 es subsupertripartientes de 7, 5 es subtriplus sesquiquintus de 16 y 5 es subquintus superquadripartientes de $29^{21}$.

\section{LOS FUNDAMENTOS MATEMÁTICOS DE LA RITMOMAQUIA}

Pasemos ahora a considerar la descripción física del tablero y de las piezas que constituyen el juego de la ritmomaquia. Las indicaciones de Boissière sobre el aspecto y las dimensiones del tablero no son estrictas. Propone un tablero rectangular dividido en casillas cuadradas iguales. Ha de tener 8 casillas de ancho, mientras que la longitud debe ser 10 como mínimo, aunque afirma que la ideal es 16 para que el producto de números pueda realizarse mejor ${ }^{22}$. Si consultamos otras fuentes confirmaremos que las dimensiones del tablero más utilizadas fueron 8x16. Respecto a la coloración de las casillas tampoco hay unanimidad, unos dibujan casillas en blanco y otros alternan casillas blancas con negras, al igual que en el tablero del

\footnotetext{
${ }^{21}$ Esta clasificación es un extracto de BoETHIUS, De Institutione Arithmetica. 1, 21 a 31.

${ }^{22}$ «Apreste un tablier pour le moins dix quarreaux ou espaces en longeur, \& s'il u en a 16 , fera meilleur d'autant que la multiplication des nombres se pourra meieux faire, $\&$ aura le dict tablier en largeur seulement huict quarreaux ou espaces» (BOISSIÈRE, 1556, p. 5 r.).

«Anuario de Estudios Medievales», 34/1 (2004), pp. 279-306.- ISSN 0066-5061.
} 
ajedrez. No parece haber una razón poderosa para decidirse por una u otra versión, quizás la claridad en la representación de las piezas en los grabados hizo que la mayoría de los textos optaran por tableros blancos.

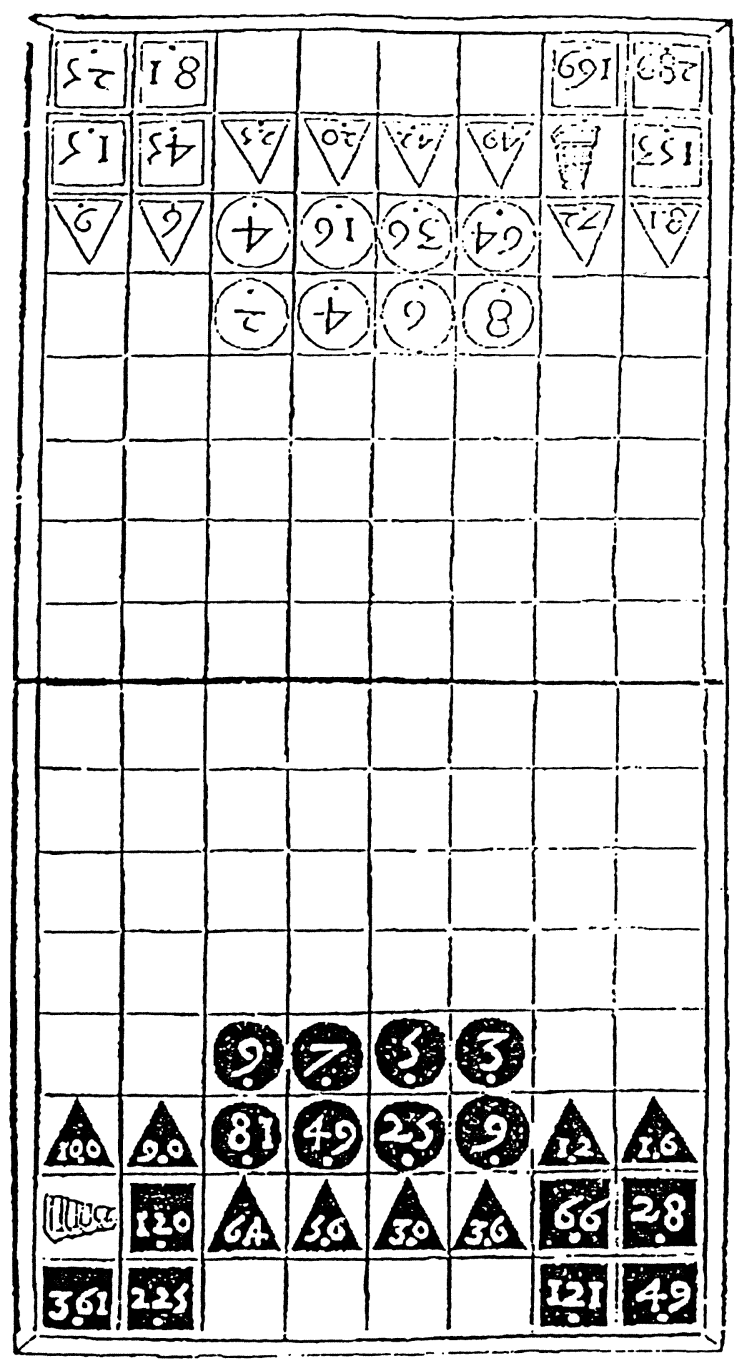

«Anuario de Estudios Medievales», 34/1 (2004), pp. 279-306.- ISSN 0066-5061. 
Respecto a las piezas, éstas deberán ser 48: 16 fichas redondas, 16 fichas con forma de triángulos equiláteros y 16 fichas cuadradas. Como es un juego para dos, cada contrincante dispone de 24 fichas, 8 de cada tipo. Las 24 de un jugador serán de color blanco y las del otro de color negro. La figura adjunta, tomada de Boissière, reproduce la distribución de las piezas en el tablero, así como los respectivos valores numéricos que cada una de ellas lleva marcado. Las fichas blancas tienen los siguientes valores numéricos: redondas $(2,4,4,6,8,16,36,64)$; triangulares $(6,9,20,25,42,49,72$, $81)$; cuadradas $(15,25,45,81,91,153,169,289)$. Las fichas negras los siguientes: redondas $(3,5,7,9,9,25,49,81)$; triangulares $(12,16,30,36$, $56,64,90,100)$; cuadradas $(28,49,66,120,121,190,225,361)$. Existen dos piezas especiales, el 91 de las blancas y el 190 de las negras, son las pirámides. Trataremos de ellas más adelante, de momento las consideraremos como cuadrados, ya que la base de las mismas lo es y además, así, se mantiene la igualdad en el número de fichas de cada forma. No todas las fuentes clásicas coinciden en la colocación de las piezas sobre el tablero, pero en lo que si existe unanimidad absoluta es en el valor numérico grabado en las piezas y en la forma de la ficha que le corresponde. Al lector actual le sorprenderá, sin duda, la sucesión de valores numéricos que aparecen en el juego, pues no parecen guardar entre si una relación numérica sencilla que pueda asociar con progresiones aritméticas o geométricas, que son los tipos de sucesiones presentadas con más frecuencia en los programas escolares contemporáneos.

A continuación, describiremos una interpretación de la generación de estos números que difiere ligeramente de la propuesta por otros autores, pero creemos que es la más coherente con la doctrina de Boecio. Se basa en cuatro hipótesis: 1) la construcción de los valores debe basarse en una aplicación estricta de la teoría de las proporciones entre números de Boecio; 2) esta aplicación debe tener en cuenta la compleja terminología empleada por ese autor; 3) la ritmomaquia pretende ser una ejemplificación didáctica de esa teoría; 4) la colocación de las piezas no es relevante, pero si lo es su forma, que debe responder a las premisas anteriores.

De acuerdo a estas bases proponemos la siguiente interpretación. Los 48 números del juego son generados, una vez descartadas el 0 y el $1^{23}$, a partir

\footnotetext{
${ }^{23}$ Los matemáticos clásicos no consideraban el uno como número, pues para ellos un número era una colección de unidades: "numerus est unitatem collectio» (BOETHIUS, De Institutione Arithmetica, 1, 3, 2).
}

«Anuario de Estudios Medievales», 34/1 (2004), pp. 279-306.- ISSN 0066-5061. 
de las restantes ocho cifras: los cuatro primeros impares $(3,5,7,9)$ para un equipo, y los cuatro primeros pares $(2,4,6,8)$ para el otro. Si colocamos estos ocho dígitos encabezando sendas columnas, como aparece en las dos tablas que se acompañan, tendremos ocho cadenas de números que tienen la característica de que cada uno está relacionado con el le precede y también con el que le sigue.

\begin{tabular}{||c||c||c||c||c||}
\hline \multirow{2}{*}{$\begin{array}{c}\text { fichas redondas } \\
\text { multiplex }\end{array}$} & $\mathbf{2}$ & $\mathbf{4}$ & $\mathbf{6}$ & $\mathbf{8}$ \\
\cline { 2 - 5 } & $2 * 2=\mathbf{4}$ & $4 * 4=\mathbf{1 6}$ & $6 * 6=36$ & $8 * 8=64$ \\
\hline $\begin{array}{c}\text { fichas triangulares } \\
\text { superparticularis }\end{array}$ & $(1+1 / 2) 4=\mathbf{6}$ & $(1+1 / 4) 4=\mathbf{2 0}$ & $(1+1 / 6) 36=\mathbf{4 2}$ & $(1+1 / 8) 64=\mathbf{7 2}$ \\
\cline { 2 - 5 } & $(1+1 / 2) 6=9$ & $(1+1 / 4) 20=\mathbf{2 5}$ & $(1+1 / 6) 42=49$ & $(1+1 / 8) 72=\mathbf{8 1}$ \\
\hline $\begin{array}{c}\text { fichas cuadradas } \\
\text { superpartiens }\end{array}$ & $(1+2 / 3) 9=\mathbf{1 5}$ & $(1+4 / 5) 25=\mathbf{4 5}$ & $(1+6 / 7) 49=91$ & $(1+8 / 9) 81=\mathbf{1 5 3}$ \\
\cline { 2 - 5 } & $(1+2 / 3) 15=\mathbf{2 5}$ & $(1+4 / 5) 45=\mathbf{8 1}$ & $(1+6 / 7) 91=\mathbf{1 6 9}$ & $(1+8 / 9) 153=\mathbf{2 8 9}$ \\
\hline
\end{tabular}

\begin{tabular}{|c|c|c|c|c|}
\hline \multirow{2}{*}{$\begin{array}{l}\text { fichas redondas } \\
\text { multiplex }\end{array}$} & 3 & 5 & 7 & 9 \\
\hline & $3 * 3=9$ & $5 * 5=25$ & $7 * 7=49$ & $9 * 9=81$ \\
\hline \multirow{2}{*}{$\begin{array}{l}\text { fichas triangulares } \\
\text { superparticularis }\end{array}$} & $(1+1 / 3) 9=12$ & $(1+1 / 5) 25=30$ & $(1+1 / 7) 49=56$ & $(1+1 / 9) 81=90$ \\
\hline & $(1+1 / 3) 12=16$ & $(1+1 / 5) 30=36$ & $(1+1 / 7) 56=64$ & $(1+1 / 9) 90=100$ \\
\hline \multirow{2}{*}{$\begin{array}{l}\text { fichas cuadradas } \\
\text { superpartiens }\end{array}$} & $(1+3 / 4) 16=28$ & $(1+5 / 6) 36=66$ & $(1+7 / 8) 64=120$ & $(1+9 / 10) 100=190$ \\
\hline & $(1+3 / 4) 28=49$ & $(1+5 / 6) 66=121$ & $(1+7 / 8) 120=\mathbf{2 2 5}$ & $(1+9 / 10) 190=361$ \\
\hline
\end{tabular}


La forma de las fichas corresponde a los tres tipos básicos de relaciones numéricas que hemos visto en Boecio al tratar las cantidades maioris: las redondas para los multiplex, las triangulares y las cuadradas para las dos especies simples, es decir, los superparticularis y los superpartiens. Los otros dos tipos de especies complejas no se incluyen, probablemente porque proporcionarían valores numéricos excesivamente altos.

Las tablas que acabamos de presentar proporcionan una explicación de cómo pueden generarse los números de la ritmomaquia a partir de una serie básica y utilizando la teoría de relación entre números descrita en Boecio. Pero esta presentación no es la que vería un alumno de una escuela monástica medieval. En esa época el simbolismo matemático que hemos empleado le sería completamente desconocido ${ }^{24}$. Por ello proponemos a continuación una versión de las mismas tablas, pero utilizando la terminología empleada en la época.

${ }^{24} \mathrm{El}$ simbolismo matemático actual comenzó a introducirse a partir del siglo XVI como resultado de las aportaciones de autores como Vieta, Widmann, Harriot, Stevin, Oughtred y otros. Véase J. REY PASTOR; J. BABINI, Historia de la Matemática. Barcelona: Gedisa, 1985, vol. II, pp. 18 y ss.

«Anuario de Estudios Medievales», 34/1 (2004), pp. 279-306.- ISSN 0066-5061. 


\begin{tabular}{|c|c|c|c|c|}
\hline \multirow{2}{*}{$\begin{array}{l}\text { fichas } \\
\text { redondas } \\
\text { multiplex }\end{array}$} & $\begin{array}{c}2 \\
\text { duo }\end{array}$ & $\begin{array}{c}4 \\
\text { quattuor }\end{array}$ & $\begin{array}{c}6 \\
\operatorname{sex}\end{array}$ & $\begin{array}{c}8 \\
\text { octo }\end{array}$ \\
\hline & 4 duplus de 2 & 16 quadruplusde4 & 36 sextuplus de 6 & 64 octuplus de8 \\
\hline \multirow{2}{*}{$\begin{array}{c}\text { fichas } \\
\text { triangulares } \\
\text { super- } \\
\text { particularis }\end{array}$} & $\begin{array}{c}6 \text { de } 4 \\
\text { sesquialter }\end{array}$ & $\begin{array}{c}20 \text { de } 16 \\
\text { sesquiquartus }\end{array}$ & $\begin{array}{c}42 \text { de } 36 \\
\text { sesquisextus }\end{array}$ & $\begin{array}{c}72 \text { de } 64 \\
\text { sesquiocta }\end{array}$ \\
\hline & $\begin{array}{c}9 \text { de } 6 \\
\text { sesquialter }\end{array}$ & $\begin{array}{c}25 \text { de } 20 \\
\text { sesquiquartus }\end{array}$ & $\begin{array}{c}49 \text { de } 42 \\
\text { sesquisextus }\end{array}$ & $\begin{array}{c}81 \text { de } 72 \\
\text { sesquiocta }\end{array}$ \\
\hline \multirow{2}{*}{$\begin{array}{c}\text { fichas } \\
\text { cuadradas } \\
\text { super- } \\
\text { partiens }\end{array}$} & $\begin{array}{c}15 \text { de } 9 \\
\text { superbi- } \\
\text { partientes }\end{array}$ & $\begin{array}{l}45 \text { de } 25 \\
\text { superquadri- } \\
\text { partientes }\end{array}$ & $\begin{array}{c}91 \text { de } 49 \\
\text { supersesqui- } \\
\text { partientes }\end{array}$ & $\begin{array}{l}153 \text { de } 81 \\
\text { superocti- } \\
\text { partientes }\end{array}$ \\
\hline & $\begin{array}{l}25 \text { de } 15 \\
\text { superbi- } \\
\text { partientes }\end{array}$ & $\begin{array}{c}\mathbf{8 1} \text { de } 45 \\
\text { superquadri- } \\
\text { partientes }\end{array}$ & $\begin{array}{c}169 \text { de } 91 \\
\text { supersesqui- } \\
\text { partientes }\end{array}$ & $\begin{array}{l}289 \text { de } 153 \\
\text { superocti- } \\
\text { partientes }\end{array}$ \\
\hline
\end{tabular}

\begin{tabular}{|c|c|c|c|c|}
\hline \multirow{2}{*}{$\begin{array}{l}\text { fichas } \\
\text { redondas } \\
\text { multiplex }\end{array}$} & $\begin{array}{c}3 \\
\text { tres }\end{array}$ & $\begin{array}{c}5 \\
\text { quinque }\end{array}$ & $\begin{array}{c}7 \\
\text { septem }\end{array}$ & $\begin{array}{c}9 \\
\text { novem }\end{array}$ \\
\hline & 9 triplus de 3 & 25 quincuplus de 5 & 49 septuplus de 7 & 81 nonuplusde9 \\
\hline \multirow{2}{*}{$\begin{array}{c}\text { fichas } \\
\text { triangulares } \\
\text { super- } \\
\text { particularis }\end{array}$} & $\begin{array}{c}12 \text { de } 9 \\
\text { sesquitertius }\end{array}$ & $\begin{array}{c}30 \text { de } 25 \\
\text { sesquiquintus }\end{array}$ & $\begin{array}{c}56 \text { de } 49 \\
\text { sesquiseptimus }\end{array}$ & $\begin{array}{c}90 \text { de } 81 \\
\text { sesquinonis }\end{array}$ \\
\hline & $\begin{array}{c}16 \text { de } 12 \\
\text { sesquitertius }\end{array}$ & $\begin{array}{c}36 \text { de } 30 \\
\text { sesquiquintus }\end{array}$ & $\begin{array}{c}\mathbf{6 4} \text { de } 56 \\
\text { sesquiseptimus }\end{array}$ & $\begin{array}{c}100 \text { de } 90 \\
\text { sesquinonis }\end{array}$ \\
\hline \multirow{2}{*}{$\begin{array}{c}\text { fichas } \\
\text { cuadradas } \\
\text { super- } \\
\text { partiens }\end{array}$} & $\begin{array}{l}28 \text { de } 16 \\
\text { supertri- } \\
\text { partientes }\end{array}$ & $\begin{array}{c}66 \text { de } 36 \\
\text { superquinti- } \\
\text { partientes }\end{array}$ & $\begin{array}{l}120 \text { de } 64 \\
\text { superseptimi- } \\
\text { partientes }\end{array}$ & $\begin{array}{l}190 \text { de } 100 \\
\text { supernoni- } \\
\text { partientes }\end{array}$ \\
\hline & $\begin{array}{l}49 \text { de } 28 \\
\text { supertri- } \\
\text { partientes }\end{array}$ & $\begin{array}{c}121 \text { de } 66 \\
\text { superquinti- } \\
\text { partientes }\end{array}$ & $\begin{array}{l}225 \text { de } 120 \\
\text { superseptimi- } \\
\text { partientes }\end{array}$ & $\begin{array}{c}361 \text { de } 190 \\
\text { supernoni- } \\
\text { partientes }\end{array}$ \\
\hline
\end{tabular}


De este modo se nos hace patente una regularidad que con el simbolismo actual no es evidente: los ocho dígitos que encabezan las columnas determinan el tipo de fracción que se añade en cada caso y también la denominación que Boecio da al número generado. Así, en los superparticularis, la fracción unitaria que se suma al número tomado como referencia tiene como denominador el dígito que encabeza la columna, lo que implica que proporciona también el lexema a la palabra latina que determina el superparticularis. Del mismo modo, el nombre latino del superpartiens viene dado por el numerador de la fracción que se añade al número con el que se compara; este numerador es precisamente el dígito que inicia la columna. Es decir, que todos los números derivados de los ocho iniciales llevan en su nombre latino el «recuerdo» del número del que parten. Esta regularidad, sin duda buscada, por el autor o autores del juego, proporcionaría una norma nemotécnica sencilla a todo aquel que deseara construir una ritmomaquia.

Las características del diseño explicarían también, por que el juego era sólo accesible a los «conocedores» de la aritmética de Boecio, es decir, a los estudiantes de las escuelas monacales y a la élite culta de la Edad Media.

La ritmomaquia es un instrumento didáctico muy útil para explicar la relación entre números. En sus fichas están ejemplarizadas las tres categorías principales de maioris, pero también pueden servir para mostrar las categorías básicas de minoris: los subsuperpartiens, los subsuperparticularis y los submultiplex. Basta con invertir el proceso, comenzar por los números que están en la base y seguir hasta la cabecera. Observemos que los números de la base muestran una peculiaridad que volvemos a encontrar en otras situaciones del juego y que no es, por tanto, casual: son todos ellos cuadrados perfectos.

Existen otras muchas relaciones notables entre los 48 números que intervienen en el juego. Nosotros sólo indicaremos otras dos, por su importancia en el desarrollo de las partidas. La primera resulta de comprobar que no todos los números que aparecen en el tablero son distintos. Hay algunos que se repiten (con fichas de igual o distinta forma). Son: 9, 16, 25, $36,49,64$ y 81 . Se trata de cuadrados perfectos, precisamente aquellos que corresponden a los números naturales del $3 \mathrm{al} 9$. El segundo hecho notable se aprecia al comprobar que, a pesar de que un equipo comienza con cuatro números pares y el otro con cuatro impares, al final, una vez generados todos los números, existe la misma cantidad de números pares que de impares en ambos equipos. 
Tratemos ahora brevemente de las dos fichas especiales, que todas las versiones de la ritmomaquia incluyen $\mathrm{y}$, además siempre asociadas a los valores 91 del equipo par y 190 del impar. Hacen sin duda referencia a un tipo de números, los llamados números piramidales, a los que los matemáticos pitagóricos y neopitagóricos, como Nicómaco o Boecio, eran sumamente aficionados. Buscando la correlación entre orden físico y orden numérico, crearon una serie de números vinculados a figuras del plano (polígonos) y a cuerpos geométricos (poliedros). Tenemos así distintos tipos de números poligonales (triangulares, cuadrangulares o cuadrados, etc.) y números poliédricos (piramidales, cúbicos, etc.). En la ritmomaquia, como juego didáctico que es, hallamos ejemplos representativos de estos números. Un ejemplo importante de número poligonal, los números cuadrados $(2,4,9,16$, $25,36,49,64, \ldots)$, ya los hemos encontrado ampliamente representados en el tablero. También están representados los números poliédricos a través de los números piramidales. Los números piramidales se obtienen como suma de números cuadrados consecutivos, cada uno de los cuales constituye un piso de una pirámide (escalonada) de base cuadrangular. 91 sería, entonces, el sexto número piramidal $91=1+4+9+16+25+36$. El 190 se puede también representar geométricamente como una pirámide, pero truncada, la denominaban tricurta por faltarle los tres primeros pisos $(190=16+25+$ $36+49+64)^{25}$. Como existen fichas de formas diferentes para los nueve primeros números cuadrados, en algunas versiones del juego, a las pirámides se las considera físicamente compuestas por la yuxtaposición de distintas piezas, este hecho otorgará a las pirámides los atributos de ataque y de vulnerabilidad propios de los tres tipos de fichas.

\section{SOBRE LAS REGLAS DE JUEGO Y SUS VARIANTES}

No hay un total acuerdo entre las distintas versiones de la ritmomaquia sobre las reglas del juego. Nosotros seguiremos a Boissière indicando sólo algunas cuestiones donde las divergencias son más notables. Comenzaremos por decir, que una vez situadas las piezas en la posición inicial (la propuesta por Boissière aparece en la ilustración), la partida la inicia siempre el bando blanco, es decir, el de los pares; de esta manera se intenta compensar

\footnotetext{
${ }^{25}$ «...mais ceste pyramide est persupposée ainsi coupée qu'ils s'en faille 3 degrez ou bases qu'elle ne parvienne lusques à l'unité: \& par aisi est dite tricurte» (BOISSIĖRE, 1556, p. 15 r.-v.).

«Anuario de Estudios Medievales», 34/1 (2004), pp. 279-306.- ISSN 0066-5061.
} 
la pequeña ventaja que tienen las impares a la hora de realizar las primeras combinaciones numéricas. El jugador que tenga el turno realizará un movimiento con una de sus fichas.

Cada tipo de ficha tiene un movimiento diferente, caracterizado por la dirección y la longitud del salto. Las fichas circulares se desplazan sólo ortogonalmente, las triangulares sólo diagonalmente y las cuadradas ortogonal y diagonalmente. Las circulares se mueven dos casillas, las triangulares tres y las cuadradas cuatro. En todos los casos se contabilizan la casilla de partida y la de llegada ${ }^{26}$. Las pirámides al considerarse constituidas por los tres tipos de fichas pueden realizar cualquiera de los tres movimientos, a criterio del jugador. Todas las fichas pueden moverse sin limitaciones, excepto las que establecen los lados del tablero y las restantes fichas. Es lógico que no puedan colocarse en casillas ya ocupadas, pero no está claro en las distintas versiones, si es posible o no saltar por encima de fichas.

Pasaremos ahora a considerar las cuatro maneras posibles de capturar fichas al contrario. Todas ellas se presentan una vez que el jugador que tiene el turno realiza su movimiento. Según sea la posición alcanzada por la ficha movida y la situación de las restantes, tanto propias como del contrario, llevará a cabo, si ello es posible, la captura. Una vez realizada ésta y la ficha contraria es retirada del tablero, la ficha atacante se mantiene en su posición sin ocupar el lugar de la atacada.

La captura por encuentro se producirá cuando una pieza pueda ocupar en un movimiento la casilla ocupada por una pieza enemiga de igual valor. Boissière advierte que si en su turno un jugador olvida retirar una pieza del contrario, en su siguiente turno podrá todavía retirarla (si lo desea), pero estará entonces obligado a realizar el movimiento y ocupar la casilla de la retirada.

La captura por emboscada se produce cuando dos o más piezas de un bando se encuentran en una posición tal que en su siguiente movimiento pueden ocupar la casilla de una pieza contraria, y la suma o diferencia de sus valores iguala el de la pieza del contrincante.

La captura por asalto se dará cuando el valor de una pieza multiplicado o dividido por el número de casillas que hay entre ella y una pieza enemiga en la dirección de su movimiento, iguala su valor. No queda claro si en el

\footnotetext{
${ }^{26}{ }_{\alpha S}$ Secondement, marche la bende des quarrez de 4 en 4 pas, ou 4 loges; les triangles marchent $3 \&$ les ronds en la suivante prochaine loge, quand ie dis quatriesme loge, i entems comptant le lieu ou la table est $\&$ celui on elle va» (BOISSIĖRE, 1556, p. 24 r.).

«Anuario de Estudios Medievales», 34/1 (2004), pp. 279-306.- ISSN 0066-5061.
} 
cómputo del número de casillas, se cuentan o no las de partida y de llegada, pues los ejemplos incluidos en las distintos textos son contradictorios.

Finalmente, la captura por asedio se produce cuando las piezas de un jugador rodean una pieza del bando contrario impidiéndole moverse. No tenemos tampoco aquí unanimidad de criterios entre los distintos textos, Boissière propone bloquear las casillas ortogonales, pero de este modo, como él mismo dice, sólo son vulnerables las fichas redondas y la pirámide. Parecería pues lógico, que el número y tipo de casillas que deben bloquearse dependa de la forma de la ficha contraria, pues cada una tiene una movilidad diferente. Obviamente los lados y esquinas del tablero son buenos aliados a la hora de planear capturas por asedio.

El ataque a las pirámides tiene una peculiaridad especial derivada de su composición. En su globalidad es una pieza difícil de capturar, pero si está constituida por la superposición de fichas de diferentes formas, pueden llevarse a cabo capturas parciales, por pisos. Con cada pérdida la pirámide verá, también, reducida su movilidad y su capacidad de ataque. La pirámide que ha perdido uno o varios de sus pisos ya no puede atacar por su valor total (91 o 190) sólo por el valor individual de cada uno de las pisos restantes, e igual ocurre con su movilidad, está dependerá del tipo de fichas que conserve.

Señalemos ahora una cuestión del reglamento del juego que también ofrece opiniones diversas, según las fuentes consultadas. Se trata del destino de las piezas capturadas. Cuando el contrincante capturaba una pieza, el jugador afectado podía substituirla por otra de entre las que él había, a su vez, capturado en jugadas anteriores. De los ejemplos que describen este tipo de jugadas, no se desprende con nitidez, si la substitución debía hacerse únicamente con fichas de igual valor numérico (que como hemos visto existían en ambos bandos) o con piezas de igual forma. Boissière incluso propone, al describir el ataque a un piso de una pirámide, que, de no disponerse de otra pieza igual, podría substituirse la pieza atacada por otra cualquiera, a criterio del jugador ${ }^{27}$. Del texto no se desprende, si esta posibilidad es aplicable en el ataque a cualquier pieza del tablero o, bien, sólo se refiere a los ataques a los pisos de la pirámide, que al ser parte de una pieza singular en el juego pudieran tener también un tratamiento diferente. De hecho, el mismo

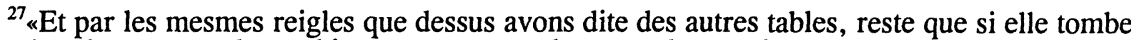
en embusche ou assault, ou bien rencontre quelque nombre egal au respect d'aucunes de ses bases, elles se rachetera (excepte le plus grande base) baillant une autre table egale à la base, au respect de laquelle elle est prinse. Et si elle nèn a point d'egale, l'adverse partie en choisira une à son plaisir» (BOISSIĖRE, 1556, p. 28 r.-29 v.).
} 
Boissière reconoce que esta regla no es compartida por otros autores, como Jacques Le Fèvre d'Estaples, en los que esta restitución de piezas sólo se daba cuando existían entre las capturadas otras de igual valor numérico ${ }^{28}$. En nuestra opinión, siguiendo la coherencia con la teoría de números descrita por Boecio, y si aceptamos limitaciones en la substitución de piezas atacadas, las únicas piezas substituibles deberían ser las que corresponden a las fichas de valores repetidos, es decir, a los números cuadrados, que son también los constituyentes de las pirámides; de este modo se destacaría a los ojos de los jugadores la importancia que este tipo de números tienen en la aritmética pitagórica.

En la ritmomaquia existen varias maneras de ganar una partida, pero, a diferencia de otros juegos en los que no suelen establecerse niveles, no todas ellas son igualmente "gloriosas». Existe una amplia tipología agrupada en torno a dos grandes categorías: victorias comunes y victorias propias.

Les victorias comunes servirían únicamente como una especie de entrenamiento de los principiantes antes de alcanzar las victorias propias, que constituirían el auténtico objetivo del juego ${ }^{29}$. Siguiendo a Boissière habría cinco tipos de victorias comunes. La victoria de cuerpo tendría en cuenta sólo el número de piezas capturadas, prescindiendo de su valor numérico. Los jugadores acordarían al comienzo de la partida un número determinado de fichas capturadas: el jugador que primero lo alcanzara sería el ganador. La victoria de bienes se refiere al valor de las piezas capturadas, ignorando cualquier otro factor. Como en la victoria anterior, los jugadores al inicio de la partida acuerdan ese valor. La victoria de proceso toma en consideración el número de cifras que aparecen en las fichas capturadas. Por ejemplo: el jugador que capturase las fichas 16, 15, 20 ganaría al que tuviera el conjunto $3,9,9,90$, pues si bien éstas últimas superan a las primeras en número y en valor, aquellas tendrían 6 cifras y éstas sólo 5. Como en los casos anteriores se requiere el acuerdo previo de los jugadores respecto a la cantidad de cifras que proporciona la victoria. La victoria de honor se basa en el número de fichas y en su valor. Finalmente, la victoria de honor y de proceso tiene en cuenta los tres aspectos: cantidad de piezas, cantidad de cifras y valor

\footnotetext{
${ }^{28}$ «On me dira quelcun qui ont traicté ce ieu [Faber Stapulensis] n'ont faict mention que la Pyramide peust racheter ses bases mineurs sinon par egales tables...” (BOISSIÈRE, 1556, p. 29 v.).

${ }^{29}{ }$ Victoires communes, sont celles lesquelles ne sont propres du ieu, ains permises pour exercer les rudes \& les rendre capables pour apres practiquer les victoires propres» (B OISSIĖRE, 1556, p. 29 r.).

«Anuario de Estudios Medievales», 34/1 (2004), pp. 279-306.- ISSN 0066-5061.
} 
numérico. Ambas victorias requieren el habitual concierto previo de los jugadores.

Las victorias propias, como su nombre indica, constituyen la esencia del juego y no dependen de la captura de fichas del contrincante, sino de la colocación de piezas en el campo contrario en una disposición regular y de modo que sus valores se encuentren en armonía, lo que equivale a decir que presenten una determinada proporción numérica. Según Boissière las victorias propias se clasifican en tres categorías: mediocres, grandes y excelentes ${ }^{30}$. Otros textos dan otros nombres, pero corresponden a idénticos contenidos.

La victoria mediocre ocurre cuando el jugador consigue disponer en el campo de su adversario tres fichas que forman una proporción cualquiera de las siguientes: aritmética, geométrica o musical. Tres números a, b, c ordenados de menor a mayor están en armonía aritmética cuando es igual la diferencia entre dos contiguos: $\mathrm{b}-\mathrm{a}=\mathrm{c}-\mathrm{b}$ (ejemplo: $8,12,16$ ); están en armonía geométrica cuando es igual el cociente entre dos contiguos: $\mathrm{b} / \mathrm{a}=$ c / b (ejemplo: 4, 6, 9) y están en armonía musical cuando el cociente entre el mayor y el menor es igual al cociente entre la diferencia de los dos mayores y los dos menores: $\mathrm{c} / \mathrm{a}=(\mathrm{c}-\mathrm{b}) /(\mathrm{b}-\mathrm{a})$ (ejemplo: $3,4,6)$. Se consigue una victoria grande cuando el jugador coloca cuatro fichas en el campo contrario de manera que formen dos armonías diferentes a la vez; es decir, aritmética y geométrica, aritmética y musical o geométrica y musical. Pero el verdadero triunfo que debe buscar el jugador experto es la victoria excelente, la cual se consigue cuando sitúa cuatro piezas en zona adversaria de manera que contengan las tres armonías a la vez: aritmética, geométrica y musical.

Existe general acuerdo en los tres tipos de victorias, pero donde surgen discrepancias derivadas de la falta de concreción en los textos, es en la disposición que deben adoptar las fichas para lograr una victoria. El mismo Boissière al comentar algunas de estas jugadas no precisa la colocación de las piezas, sólo nos dice que debe ser en campo contrario ${ }^{31}$. Algunos comentaristas modernos (MEBBEN 1996) opinan que las fichas debían situarse equidistantes entre si adoptando alguna de las tres disposiciones siguientes: en línea recta paralela a un lado del tablero, formando ángulo recto (o un triángulo, según se mire) o, si eran cuatro, situándose en los vértices de un cuadrado.

\footnotetext{
${ }^{30}$ «Nous avons trois sortes de victoires propres. A sçavoir mediocre, grande \& excellente» (BOISSIÈRE, 1556, p. 33 r.).

${ }^{31}$ «au champ de l'adversaire» (BOISSIÈRE, 1556, p. 33 v.).

«Anuario de Estudios Medievales», 34/1 (2004), pp. 279-306.- ISSN 0066-5061.
} 
No se especifica si el orden de colocación importa o no, aunque probablemente no, pues lo contrario supondría una importante dificultad añadida. Parece aceptable esta interpretación, pues se basa en una distribución geométrica regular de las fichas, lo que resulta coherente con el pensar matemático de la Escuela Pitagórica.

Debemos comentar, aunque sea muy brevemente, un hecho importante que afecta a la realización de victorias. Se trata de la posibilidad de utilizar las piezas capturadas para completar la serie de números que proporcionan una armonía. Los textos clásicos contemplan esta posibilidad en los ejemplos, pero no desarrollan las condiciones bajo las cuales se realiza. Así, no sabemos, por ejemplo, cuantas y cuales fichas pueden ser aprovechadas, bajo que circunstancias y en que posición se deben colocar. Los estudiosos del tema no han proporcionado tampoco mucha más luz sobre esta cuestión. Han comprobado que la utilización de piezas capturadas es necesaria para equilibrar las posibilidades de alcanzar victorias por parte de ambos equipos y, especialmente, para las que requieren armonías musicales (BORST 1986). También han propuesto, por razones de dinámica de juego, que no puede utilizarse una pieza capturada para completar la armonía y conseguir así una victoria (MEBBEN 1996). Para los restantes interrogantes las interpretaciones están abiertas. En nuestra opinión, este carácter abierto que tienen algunas de las reglas de la ritmomaquia era deliberado. Boissière, consciente de estas indeterminaciones, afirma que esa era precisamente una de las virtudes del juego: la libertad que tienen sus jugadores de introducir y modificar reglas a su gusto, pues los juegos que no son a gusto de los jugadores, dejan de ser juegos ${ }^{32}$.

Digamos, finalmente, que existen muchas combinaciones de grupos de tres o de cuatro piezas de la ritmomaquia que proporcionan algún tipo de armonía. Algunos autores (RICHARD, ILLMER y MEBBEN) han determinado todas las combinaciones posibles (incluyendo las fichas de ambos bandos). Dada esta multiplicidad de soluciones no nos puede extrañar que los jugadores llevaran consigo tablas con las distintas posibilidades como ayuda o inspiración en sus partidas. Precisamente uno de los objetivos de los antiguos textos era el de proporcionar al lector tablas de armonías. Aunque, lo que en

\footnotetext{
${ }^{32}$ «Au demeurant nous ne voulons pource deroger à la liberté des ioueurs, lesquels si autrement que nous veullent limiter ou determiner leurs ieux, qu'ils ne le puissent faire: Car si les ieu n'estoient au plaisir des ioueurs, ne seroient ieux, ans contraincte» (BOISSIÈRE, 1556, p. 39 r.).

«Anuario de Estudios Medievales», 34/1 (2004), pp. 279-306.- ISSN 0066-5061.
} 
verdad cabía esperar de un jugador experto era que fuera capaz de elaborar sus propias tablas, incluyendo en ellas soluciones originales, hallando en esa labor la misma satisfacción, dice Jacques Le Fèvre d'Estaples, que los cazadores que prefieren seguir y buscar sus propias presas antes que recibirlas de otros ${ }^{33}$.

\section{CONCLUSIONES}

No creemos exagerado concluir que la ritmomaquia es el primer juego didáctico de la historia de la educación matemática occidental. De la descripción de los fundamentos del juego y de sus reglas se infiere que todo el conjunto fue pensado y diseñado cuidadosamente para orientar en la instrucción y el perfeccionamiento de la aritmética desde la perspectiva neopitagórica descrita por las De Institutione Arithmetica de Boecio. Es notable la habilidad del autor o autores por elegir los números que intervienen en el juego. Partiendo de los valores más simples, los dígitos, construye un conjunto de números que representan las principales relaciones numéricas estudiadas en la época, así como también muestran el maridaje entre aritmética y geometría tan caro a los seguidores de Escuela de Pitágoras. Se consigue una perfecta adecuación entre teoría y práctica. Por su parte, los movimientos de las piezas y las normas de captura, al hacer intervenir las cuatro operaciones básicas del cálculo, favorecen la ejercitación de las mismas. Y el objetivo de la ritmomaquia, conseguir situar piezas del juego en una disposición geométrica regular y de modo que se cree una relación de proporción «bella» entre los valores numéricos, una armonía entre números, representa la culminación del saber aritmético medieval integrado dentro de un conocimiento filosófico-teológico que le da sentido: la contemplación del orden del universo a través de esa maqueta a escala que constituye la serie de números lograda por el ganador.

¿La ritmomaquia puede ser recuperada en la actualidad con una finalidad didáctica? Esta pregunta no puede ser respondida de un modo tajante, requiere diversos grados de matización. En primer lugar consideremos

\footnotetext{
${ }^{33}$ «Il y e a encore aucune comme 5, 9, 45, 81 ou bien 5, 25, 45, 225 \& autres semblables, lesqueles laisserons pour la recreation dels plus subtilz: lesquels, (comme dit Faber), a l'imitation des chasseurs plus se delectent à suyvir \& chercher leur proye, que non pas à la recevoir prinse» (BOISSIÈRE, 1556, p. 39 v.). 
el nivel docente de aplicación. Si nos referimos a la formación de profesores, el conocimiento de la ritmomaquia nos parece absolutamente conveniente, especialmente si se incorpora a programas que contemplen la historia de la educación matemática o incidan en el desarrollo de juegos y sus estrategias. En el primero de los casos sirve como excelente introducción al estudio de la enseñanza de la aritmética en el mundo medieval, pues como hemos visto, resume perfectamente sus principales contenidos; y en el segundo, al ser un juego numérico lo suficientemente complejo, permite atraer la atención de los interesados en el análisis de estrategias.

Ahora bien, si lo que se plantea es su utilización con alumnos de primaria o secundaria, entonces la respuesta no puede ser tan concluyente. Dado que, ni los temas ni los enfoques de la aritmética en nuestros programas escolares coinciden con los que se proponían en las escuelas monásticas de la Edad Media, una aplicación del juego en los mismos términos que hemos descrito en este trabajo nos parece extemporánea. Los intentos por incluir la ritmomaquia en programas escolares alemanes en algunos centros en el siglo XIX y también a principios del XX condujeron, como ya hemos comentado, al fracaso. Ha habido otras propuestas no tan ambiciosas, algunas (NEWTON, SHANNON) no han pasado de ser meras descripciones del juego y sus reglas, otras (EVANS, MEBBEN) parecen más orientadas a la recuperación del juego en si mismo que en su posible adaptación al ámbito docente.

Los elementos más característicos de la ritmomaquia no son transportables al contexto escolar actual. Si nos fijamos en la elaborada terminología latina que acompaña a las diversas categorías de relaciones entre números, comprobamos inmediatamente que se ha perdido prácticamente en su totalidad. Solamente subsisten las relaciones de múltiplos (duplo, triple, cuádruple, quíntuple, etc.) y de submúltiplos. Toda la variada tipología de números con imagen geométrica también se ha olvidado en su mayor parte, conservándose sólo algunos, como los números cuadrados y los números cúbicos, por su vinculación a los conceptos de área y volumen. Por lo que se refiere a las armonías, que hoy se prefiere denominar progresiones (aritméticas, geométricas, ...), siguen apareciendo en los currícula, pero ya no ocupan el lugar de preeminencia que tenían reservado en el medievo. De todo lo dicho se deduce, que la viabilidad didáctica de un juego basado, en buena medida, en unos conceptos no enseñados en la actualidad o sólo tratados parcialmente y con unos objetivos que han perdido la relevancia que tuvieron en otra época, resulta cuestionable y exige, en todo caso, un replanteamiento a fondo. 


\section{BIBLIOGRAFÍA BÁSICA SOBRE RITMOMAQUIA}

BAROZZI, F., Il nobilissimo et antiquissimo giuco pythagoreo nominato Rythmomachia cioe battaglia de consonanti e de numeri. Ritrovato per utilità \& solazzo delli studiosi, et al presente in lingua volgare in modo di paraphrasi composto, Venecia, G. Perchacino, 1572.

Boethius, De institutione arithmetica. Libri duo, Lipsiae, B.G. Teubneri, 1867. (Existen varias versiones modernas bilingües, como la francesa debida a Jean-Yves Guillaumin, Institution arithmétique, Paris, Les Belles Lettres, 1995 o la española de María Asunción Sánchez Manzano, Institutione arithmetica, León, Universidad de León, 2002).

BuXERIUS, C., Nobilissimus et antiquissimus ludus Phythagoreus (qui Rythmomachia nominatur) in utilitatem \& relaxationem studiosorum comparatus ad veram \& facilem propietatem \& rationem numerorum, Paris, Guilielmum Cavellat, 1554.

BOISSIÈRE, C., Le très excellent et ancien jeu Pythagorique, dict Rythmomachie, fort propre \& très utile à la récréation des esprits vertueux, pour optenir vraye \& prompte habitude en tout nombre \& proportion, Paris, Amet Breire, 1556.

Borst, A., Das mittelalterliche Zahlenkampfspiel (Supplemente zu den Sitzungsberichten der Heidelberger Akademie der Wissenschaften. Philosophischhistorische Klasse, Vol. 5), Heidelberg, Akademie der Wissenschaften, 1986.

Evans, G.E., The Rithmomachia: a Mediaeval Mathematical Teaching Aid?, «Janus», 63/4 (1976), pp. 257-273.

FABER STAPUlENSIS, J. (BOETHIUS-NemORARIUS, J.), In hoc opera contenta. In Jordanus Nemorarius arithmetica decem libris demonstrata. Musica libris demonstrata quattor. Epitome libros arithmeticos divi Severini Boetii. Rithmimachiae ludus qui pugna numerorum appellantur, Paris, D. Lauxius, 1496.

Illmer, D.; GÄDEKE, N.; Henge, E.; PfEIfFER, H.; SPICKER-BECK, M., Rhythmomachia: Ein uraltes Zahlenspiel neu entdeckt von..., München, $\mathrm{H}$. Hugendubel, 1987.

LEVER, R.; FUlKer, W., The most noble ancient, and learned Playe, called the Philosophers Game, London, J. Rowbothum, 1563.

MEBBEN, P., Rithmomachie. Ein aus dem Mittelalter überliefertes Zahlenspiel: Neu entdeckt für die Schule (Tesis doctoral) Freiburg, Pädagogische Hochschule, 1996.

MOYER, A.E., The Philosopher's Game (Reproducción íntegra del texto de LeverFulker de 1563) University of Michigan Press, 2001.

NEwTon, D.P., Rithmomachia, «Mathematics in School», 13/2 (1984), pp. 2-4.

«Anuario de Estudios Medievales», 34/1 (2004), pp. 279-306.- ISSN 0066-5061. 
NicomaChUS OF GERASA, Introduction to Arithmetic (Traducción de M.L. D'Ooge) New York, Dover, 1926.

PEIPER, R., Fortolfi Rythmomachia, «Zeitschrift für Mathematik und Physik», 25 (1880), pp. 167-198 (Contiene la versión de la ritmomaquia del manuscrito Breslau 54).

RICHARD, J.F., A new Manuscript of a Rithmomachia, «Scripta Mathematica», 9 (1943), pp. 169-183, 256-264.

RICHARD, J.F., Boissiere's Pythagorean Game, «Scripta Mathematica», 12 (1946), pp. 177-217.

RIES, A., Arithmomachia, Dresde Bibliothek, 1562. Manuscrito C-433.

SElENUS, G., Das Schach oder König-Spiel, Zürich, Olm, 1978 (Reproducción de la edición de 1616 de Leipzig, Menningen, en la que el juego de la ritmomaquia se incluye como apéndice con el título: Rythmomachia. Ein vortrefflich und uhraltes Spiel des Pythagorel).

SHANnon, A.G., Rithmomachia: a Number's Battle, «Australian Mathematics Teacher», 39/2 (1983), pp. 6-8.

SHIRWood, J., Ad reverendissimum religiosissimumque in Christo patrem ac amplissimum dominum Marcum cardinalem Sancti Marci vulgariter nuncupatum Johannis Shirvuod quod latine interpretatur Limpida Silva sedis Apostolicae protonotarii Anglici, praefatio in Epitomem de ludo arithmomachiae feliciter incipit, Rome, U. Han, 1480.

SMIth, D.E.; EATon, C., Rithmomaquia, the great medieval Number Game, «American Mathematical Monthly», 18 (1911), pp. 73-80.

SMith, D.E. , Rara Arithmetica, New York, Chelsea, 1970.

STIGTER, J., Rithmomachia: het Cijferspel, «Voordracht op het 27e Nederlandse Mathematisch Congres», Rotterdam, Erasmus Universiteit, 1991.

WAPPLER, E., Bemerkungen zur Rhythmomachie, «Zeitschrift für Mathematik und Physik», 37 (1892), pp. 1-23 (Incluye el texto de las versiones Hermanni y Wirceburgensis contenidas en el manuscrito München 14836). 